PSICOLOGÍA

IBEROAMERICANA
Psicología Iberoamericana ISSN: 1405-0943

revista.psicologia@ibero.mx

Universidad Iberoamericana, Ciudad de México México

\title{
Inidicadores de fiabilidad de la Escala de Habilidades Psicoterapéuticas Humanistas ehph en psicoterapeutas de Ciudad Juárez
}

\author{
Lozano Martínez, Jorge Ramón; Castro Valles, Alberto; Vidaña Gaytán, María Elena; González Valles, \\ María Nieves \\ Inidicadores de fiabilidad de la Escala de Habilidades Psicoterapéuticas Humanistas ehph en \\ psicoterapeutas de Ciudad Juárez \\ Psicología Iberoamericana, vol. 26, núm. 2, 2018 \\ Universidad Iberoamericana, Ciudad de México, México \\ Disponible en: http://www.redalyc.org/articulo.oa?id=133959841007
}




\title{
Inidicadores de fiabilidad de la Escala de Habilidades Psicoterapéuticas Humanistas ehph en psicoterapeutas de Ciudad Juárez
}

\author{
RELIABILITY INDICATORS OF HUMANISTIC PSYCHOTHERAPEUTIC SKILLS SCALE EHPH IN \\ PSYCHOTHERAPISTS OF CIUDAD JUAREZ
}

Jorge Ramón Lozano Martínez

Universidad Autónoma de Ciudad Juárez, México

Redalyc: http://www.redalyc.org/articulo.oa?

jorge.lozano@uacj.mx

Alberto Castro Valles

Universidad Autónoma de Ciudad Juárez, México

Maria Elena Vidaña Gaytán

Universidad Autónoma de Ciudad Juárez, México

María Nieves González Valles

Universidad Autónoma de Ciudad Juárez, México id $=133959841007$

Recepción: 17 Abril 2018

Aprobación: 19 Marzo 2019

\section{ReSUMEN:}

El objetivo del presente estudio fue diseñar y conocer la fiabilidad de la Escala de Habilidades Psicoterapéuticas Humanistas (ehph), la cual alcanzó un alpha de Cronbach de a=.865. Participaron 51 psicoterapeutas de Ciudad Juárez con estudios de posgrado y al menos un año de práctica. Se identificaron seis factores asociados a las fases del “Ciclo de la Experiencia” de la Psicoterapia Gestalt y se agruparon en cinco subescalas: Darse Cuenta, Autodeterminación, Acción, Contacto y Asimilación. El 84\% de los participantes resultó con habilidades psicoterapéuticas adecuadas y 16\% medianas. En el Darse Cuenta 65\% con habilidades adecuadas, 33\% medianas y $2 \%$ inadecuadas; en la Autodeterminación 96\%, Contacto y Acción 94\% con habilidades adecuadas; y en la Asimilación $57 \%$ habilidades adecuadas, $41 \%$ medianas y $2 \%$ inadecuadas. Se espera que la Escala de Habilidades Psicoterapéuticas Humanistas (ehph) pueda ser utilizada en procesos de formación y supervisión de psicoterapeutas como guía para desarrollar habilidades psicoterapéuticas.

Palabras ClaVe: Fiabilidad, habilidades psicoterapéuticas humanistas, psicoterapeutas, Ciudad Juárez, ciclo de la experiencia.

\section{Abstract:}

The objective of the present study was to find reliability indicators of the Humanistic Psychotherapeutic Skills Scale (ehph), which reached an alph of Cronbach of $a=.865 .51$ amongst psychotherapists from Ciudad Juarez. Six factors associated with the phases of the "Cycle of Experience" of Gestalt Psychotherapy were identified and grouped into five subscales: awareness, self-determination, action, contact and assimilation. Eighty-four percent of the participants showed adequate psychotherapeutic skills, whilst $16 \%$ showed medium skills. Results indicated that in terms of awareness $65 \%$ had adequate skills, 33\% had medium skills and $2 \%$ had inadequate skills; $96 \%$ displayed self-determination, 94\% showed adequate skills in contact and action; and in terms of assimilation 57\% showed adequate skills, $41 \%$ showed medium skills and $2 \%$ showed inadequate skills. The study shows that the Humanistic Psychotherapeutic Skills Scale (ehph) can be used in the training and supervision process of psychotherapists as a guide to developing psychotherapeutic skills.

KEYWORDs: Reliability, humanistic psychotherapeutic skills, psychotherapists, Ciudad Juarez, cycle of experience .

\section{INTRODUCCIÓN}

En los últimos años se ha puesto un especial énfasis en determinar la eficiencia en los tratamientos de la salud mental, así como la mejora continua en la calidad del servicio que se ofrece. Por lo general, el enfoque particular del psicoterapeuta es su efectividad, es decir, que sus pacientes reporten mejoría en los síntomas asociados a su motivo de consulta. Más para el mundo científico y los gobiernos el interés se centra en 
la eficacia de los tratamientos empleados, tener evidencias de cómo tratar un padecimiento y contrastar los resultados con diferentes enfoques psicoterapéuticos para determinar cuáles son más eficientes (Bados, García, \& Fusté, 2002; Case, 2011; Ferro \& Vives, 2004; oms, 2003, 2005; Paredes-Rivera, 2016).

El interés en común entre psicoterapeutas, gobierno, instituciones y comunidad es brindar el mejor servicio posible que reduzca los síntomas y aumente la calidad de vida de los pacientes con un impacto positivo a nivel personal y comunitario. Los resultados que se obtienen no dependen únicamente del procedimiento empleado, también influyen el paciente y su contexto, su personalidad, la complejidad de su padecimiento y la disposición al tratamiento, la alianza psicoterapéutica, la personalidad del psicoterapeuta, su actitud y nivel de experiencia, así como sus habilidades para realizar un trabajo psicoterapéutico (Winkler, Cáceres, Fernández, $\&$ Sanhueza, 1989). Aunque las habilidades del psicoterapeuta no son lo único que define la calidad en el servicio de la salud mental, sí influyen significativamente en los resultados (Bados \& García, 2011; Marinho, Caballo, \& Silveira, 2003; oms, 2003; SS, 2014).

Una habilidad es la capacidad para ejecutar algo con destreza (rae, 2019) y su desarrollo requiere de conocimiento sobre el tema y de práctica constante (Watson \& Tharp como se citó en Marinho et. al., 2003). Como habilidades psicoterapéuticas podemos entender las acciones que realiza un psicoterapeuta con el propósito de mejorar la salud mental de sus pacientes (Bados et. al., 2002). El desarrollo de estas habilidades inicia en la etapa formativa de especialización en psicología clínica o psicoterapia y que en México sólo se obtiene al cursar un posgrado (Sánchez-Sosa, 2012).

Según datos de un programa educativo de Maestría en psicoterapia humanista de la Universidad Autónoma de Ciudad Juárez en entrevistas con administradores, se identificó que existen 85 egresados de este posgrado inscrito en el padrón nacional de posgrados de calidad Conacyt y que en el seguimiento de egresados el $80 \%$ se encuentra ejerciendo actividades de psicoterapia con enfoque humanista. El uso de instrumentos de evaluación sobre las habilidades que se requieren para la labor psicoterapéutica sirve de guía para mantener y aumentar la eficacia del psicoterapeuta. Permite obtener retroalimentación valiosa para identificar las habilidades ya adquiridas y afianzarlas, así como elaborar estrategias de mejora para las que no han sido aún desarrolladas tanto a nivel individual como colectivo (institucional o académico). Además, son un argumento para promover una práctica profesional ética sustentada en la capacitación constante y la mejora continua. Estos instrumentos pueden ser empleados por alumnos, maestros y supervisores durante la formación académica, así como en procesos de auto-observación o supervisión institucional e incluso de manera informal entre pares, pero sí de forma comprometida.

\section{ESTADO DEL ARTE}

Existe poca literatura sobre la evaluación de habilidades psicoterapéuticas humanistas, así como de cualquier otro enfoque. Sin embargo, últimamente ha crecido el interés en investigar y hacer aportaciones documentadas sobre este tema. A continuación, se presentan algunos de estos estudios.

El Instituto Gestalt de Cleveland utiliza un cuestionario de autoanálisis para que los psicoterapeutas en formación identifiquen sus competencias, así como su estilo particular para realizar psicoterapia. Nevis define 12 habilidades características del enfoque gestáltico: 1) expresión clara, precisa, concisa y directa; 2) enfoque en el presente, "aquí y ahora"; 3) sensibilidad sensorial y consciencia corporal; 4) consciencia emocional y su expresión adecuada; 5) distinguir entre fenómenos observados de interpretación; 6) consciencia de las propias intenciones y claridad para expresarlas; 7) consciencia y respeto del momento existencial del paciente; 8) confianza en el continuum del proceso y alianza psicoterapéutica; 9) versatilidad para mostrar firmeza o suavidad de acuerdo a la situación; 10) respetar y resolver situaciones emocionales con el paciente; 11) ser atractivo sin pretender ser carismático; y 12) consciencia de la creatividad y trascendencia de su trabajo. El cuestionario describe diversas acciones asociadas a habilidades para que el psicoterapeuta en formación identifique si las utiliza o no en su práctica (Castanedo, 1999). 
Marinho et al. (2003) mencionan que las habilidades del enfoque Cognitivo-Conductual se basan en la escucha y la demostración de comprensión para establecer una relación terapéutica competente. Estas son: actitud de respeto y aceptación; mantener la atención en el paciente; empatizar; presentar elementos corporales apropiados; elementos paralingüísticos adecuados; realizar preguntas abiertas; parafrasear; reflejar sentimientos; mostrar comprensión del contexto; y manejar conductas de escape/evitación del paciente. Los autores enfatizan que la capacitación en este enfoque ha priorizado el aprendizaje de técnicas y no el desarrollo de las habilidades mencionadas; y que el desarrollo de éstas permite mejorar la eficiencia en la práctica.

El curso de formación básica de psicoterapeutas -previo a la especialización en posgrado de cualquier enfoque- utilizado por Beitman y Yue (2004) consiste en el desarrollo de habilidades de: 1) escucha; 2) intenciones y respuestas verbales del psicoterapeuta; 3) alianza psicoterapéutica; 4) razonamiento inductivo para determinar causas disfuncionales de acuerdo al enfoque psicoterapéutico utilizado; y 5) estrategias de cambio. El entrenamiento se realiza por fases para ir afianzando gradualmente las habilidades anteriores. Utilizan diversos formularios autoaplicados tanto por el psicoterapeuta como por el paciente, al inicio, durante y posterior al curso de formación. Estos formularios son de tipo Likert, preguntas guiadas para responder de forma abierta-cualitativa o de acuerdo con listados de respuestas clasificadas.

Casas (2011) realizó una tesis llamada Técnicas expresivas: Su integración en las prácticas profesionales de los psicólogos egresados de la Universidad de la República. Su objetivo fue describir el uso de técnicas expresivas en el desempeño profesional de psicólogos egresados entre 1985 y 2000 de la Universidad de la República de Montevideo, Uruguay. El marco teórico que dio soporte a su estudio fue la creación-expresión desde las perspectivas del psicoanálisis, la teoría jungiana, la terapia Gestalt y el conductismo. Aplicó una mezcla de metodología cuantitativa-cualitativa de tipo exploratorio descriptivo. Se realizaron entrevistas semiestructuradas a especialistas además de la aplicación de un cuestionario a una muestra intencional no probabilística para recabar información primaria. A partir de los resultados obtenidos fue posible elaborar un perfil de las prácticas, tipo de formación y ubicación laboral de los psicólogos que usan técnicas expresivas. Concluye que el empleo adecuado de técnicas expresivas sin importar el enfoque psicoterapéutico puede responder a la demanda de atención de salud mental en la comunidad.

En el articulo "Habilidades Terapéuticas", Bados y García (2011) afirman que el logro de los objetivos psicoterapéuticos no reside en la aplicación de técnicas sino en la creatividad y la sensibilidad para aplicarlas. Para tener acceso a éstas se requiere de: 1) actitudes básicas de relación como la escucha activa, empatía, aceptación incondicional y autenticidad; 2) características básicas de la relación como la cordialidad, competencia técnica, confianza, atracción y grado de directividad; 3) habilidad es escucha en la comunicación como lo es clarificar, parafrasear, reflejar y sintetizar; 4) habilidades de expresión en la comunicación entre las que se encuentran preguntar, confrontar, interpretar e informar; 5) habilidades para entrevistar; 6) adaptación de la intervención a la personalidad del paciente para definir problemas, comunicar información, motivación y expectativas, considerar las variables demográficas del paciente, el tipo de problema y enfermedades o déficits físicos que lo incapaciten; 7) equilibrar la relación; 8) manejar dificultades en el proceso; y 9) elaborar estrategias para mejorar las intervenciones.

Case (2011) elaboró un prototipo con las características ideales de un proceso psicoterapéutico con enfoque Gestalt utilizando un Proceso de Psicoterapia Q-Set (pqs) con un grupo de expertos en psicoterapia Gestalt. Se incluyen comportamientos del terapeuta, del paciente y de interacción entre éstos. El prototipo se puede utilizar como base para definir las habilidades psicoterapéuticas propias de enfoque gestáltico. Entre los comportamientos del psicoterapeuta con enfoque gestáltico se incluyen: 1) aceptar sin prejuicios a su paciente; 2) sensibilidad, empatía y sintonía con los sentimientos del paciente; 3) apoyar al paciente; 4) comunicarse de forma clara y coherente; 5) percibir de manera precisa el proceso psicoterapéutico; 6) evidenciar el comportamiento no verbal; 7) identificar los patrones conductuales recurrentes; 8) comentar los cambios de estado emocional; 9) evidenciar los sentimientos que el paciente considera inaceptables; 10) centrarse en la relación presente; 11) asociar el tipo de relación que se da en el proceso psicoterapéutico 
con otras relaciones; 12) dar importancia a las relaciones interpersonales; 13) enfatizar sentimientos que se presenten en el proceso; 14) ofrecer diferentes perspectivas a las experiencias o eventos del paciente; 15) alentar a nuevas formas de comportamiento; 16) reafirmar, reformular y aclarar la comunicación; 17) explorar sueños y/o fantasías; 18) priorizar el presente; 19) centrarse en el diálogo.

Fogarty (2015) presentó avances sobre la construcción de la Escala de Fidelidad de la Terapia Gestalt, la cual es el tema central de su investigación de tesis doctoral. Emplea el método Delphi para obtener un consenso de expertos sobre los comportamientos del psicoterapeuta con formación en Gestalt. Es una escala que identifica el uso o no uso de 20 comportamientos característicos de este enfoque. Puede ser autoaplicada y/o aplicarse por otros observadores para establecer un contraste de perspectivas. Incluye una pregunta más para identificar factores inusuales que determinen la no pertinencia del uso de terapia Gestalt en el proceso. En este consenso los expertos determinaron que el objetivo central del enfoque gestáltico es el "darse cuenta" (consciencia inmediata) y el "darse cuenta del darse cuenta" (consciencia reflexiva) mediante la práctica de 7 conceptos básicos: 1) relación dialogal; 2) trabajar en el "aquí y ahora"; 3) práctica fenomenológica; 4) trabajar la consciencia corporal; 5) práctica sensible al campo (organismo-entorno); 6) trabajar con el proceso de contacto; y 7) uso de una actitud experimental.

Las anteriores aportaciones se enfocan en determinar cuáles son las habilidades psicoterapéuticas que pueden contribuir a prácticas eficientes, sin embargo, no alcanzan a proponer un instrumento que las evalué. Por lo que surge la siguiente pregunta de investigación: ¿̨e podrá contar con un instrumento de evaluación de habilidades psicoterapéuticas humanistas fiable como guía para la formación y supervisión de psicoterapeutas que posibilite la mejora continua de su servicio?

\section{EL PARADIGMA HUMANISTA}

La psicoterapia humanista promueve el desarrollo del potencial humano para que los pacientes se responsabilicen de su vida, libre de dependencias. En contraste, en las psicoterapias con enfoque psicodinámico o conductista se depende del psicoterapeuta para eliminar patologías. La psicología bumanista es simultáneamente un movimiento tanto teórico como social que prioriza las experiencias de los seres humanos, sus valores y propósitos, así como sus significados, además de promover el desarrollo personal y el cambio (Castanedo \& Mungía, 2011).

\section{Ciclo de Autorregulación Organísmica}

Los principios teóricos de la psicoterapia Gestalt son la Teoría de la Gestalt (que incluye las Teorías de Figura y Fondo, del Círculo de la Forma, del Aprendizaje, y de Campo), el holismo cuerpo-mente-ambiente, la autorregulación organísmica y la Teoría Paradójica del Cambio (Perls, Hefferline, \& Goodman, 2003; Taragano, 1974; Yontef, 1995). Su metodología se basa en promover la autorregulación organísmica mediante un diálogo existencial que integra el Darse Cuenta, el análisis fenomenológico y la Teoría Paradójica del Cambio de Beisser (Yontef, 1995).

Nuestro método consiste, por lo tanto, en lo siguiente: mostrar que, en las condiciones en las que el observador tiene la experiencia, éste debe mantener su opinión y, después, mediante el empleo de la consciencia inmediata en estas condiciones restrictivas, facilitamos que surja (en él y en nosotros mismos) un juicio mejor... (Perls et al., 2003, p. 26). La finalidad del tratamiento no es resolver la mayoría de los complejos o liberar ciertos reflejos, sino llegar a un punto tal en la técnica de la consciencia inmediata de sí mismo que el paciente pueda sin ayuda... la curación sólo pude venir de uno mismo (en el entorno) (Perls et al., 2003, p. 32).

El objetivo central de la psicoterapia Gestalt es "darse cuenta” (awareness) como estructura y como proceso (Fogarty, Bhar, Theiler, \& O'Shea, 2016; Perls, 1975; Perls et al., 2003; Yontef, 1995).

El darse cuenta es el proceso de estar en contacto alerta con el evento más importante del campo individuo/ ambiente, con total apoyo senso-motor, emocional, cognitivo y energético. Un continuo e ininterrumpido 
continuum del darse cuenta lleva a un “iAjá!”, la captura inmediata de la unidad obvia de elementos dispares en el campo. Así, se crean totalidades nuevas y significativas. Darse cuenta es en sí mismo una integración del problema (Yontef, 1995, p. 191-192). El “darse cuenta” per se -por y de sí mismo- puede ser curativo. Porque con un awareness pleno uno se da cuenta de esta autoregulación organísmica, uno puede permitir que el organismo se haga cargo sin interferir, sin interrumpir, podemos fiarnos de la sabiduría del organismo. Todo está basado en el darse cuenta. El darse cuenta es la única base de conocimiento, de la comunicación... (Perls, 1974, p. $28 \& 56$ ).

El análisis fenomenológico se emplea para explorar el proceso de "darse cuenta" (Perls, 1975; Yontef, 1995), y comprender cómo se está en una situación dada, basándose en lo obvio o lo que revela la situación (incluyendo el organismo y el ambiente) más que lo que el psicoterapeuta como observador pudiera interpretar. Una exploración fenomenológica implica describir detallada y claramente lo que es, y no lo que debería o podría ser, ni lo que fue o será (Yontef, 1995). La Teoría Paradójica del Cambio establece que: "El cambio se produce cuando uno se convierte en lo que es, no cuando trata de convertirse en lo que no es" (Beisser como se citó en Fagan \& Sheperd, 1973, p. 82). Al estimular el contacto con lo que la persona y su ambiente realmente se posibilita un desarrollo natural de crecimiento del paciente definido por su autorregulación organísmica y no un cambio forzado por las expectativas del psicoterapeuta o de cualquier teoría (Yontef, 1995).

Para analizar el funcionamiento de la Autorregulación Organísmica, Zinker (2003) desarrolló un esquema conocido como "Ciclo de la Autorregulación del Organismo" (cao) o "Ciclo de la Experiencia". Está compuesto por seis fases: Sensación, Consciencia, Movilización de Energía y Excitación, Acción, Contacto y Retirada. En la fase de Sensación existen experiencias indiferenciadas de estímulos orgánicos, necesidades fisiológicas, sentimientos corporales, ideas, imágenes y percepciones del ambiente. Durante la segunda fase, el proceso de darse cuenta atiende y organiza las sensaciones para tomar consciencia de la sensación más relevante (antes indiferenciada) convirtiéndose en la figura de mayor interés sobre un fondo, dándole un significado a la experiencia y poder comprender qué es lo que necesita el organismo y cómo satisfacerlo. La Movilización de Energía y Excitación es un proceso que inicia con la visualización del posible satisfactor y las posibles acciones para obtenerlo, se ensayan y analizan mentalmente las diferentes posibilidades para tomar la decisión que mejor responda a la situación del campo organismo-ambiente. Simultáneamente a este proceso de elección, el organismo moviliza la energía disponible, se excita y se carga de ésta para realizar las acciones correspondientes. La fase de Acción incluye cualquier actividad física o expresión que se realice para acercarse y contactar al posible satisfactor. El Contacto es un proceso que involucra a todo el organismo en el que abre sus fronteras para intercambiar energía con el posible satisfactor, discriminando y asimilando lo que sea conveniente o nutritivo de la novedad del momento. En la Retirada, el organismo retrocede, reflexiona para asimilar la experiencia completa generando crecimiento; establece nuevos límites y aumenta su autonomía. En la realidad, las fases están sucediendo todo el tiempo y se intercalan en un solo momento, sin embargo, el esquema de Zinker las presenta en forma secuencial para su comprensión y facilitar su identificación permitiendo hacer un diagnóstico fenomenológico y orientar las intervenciones del psicoterapeuta (Castanedo, 1999; Kepner, 1999; Zinker, 2003).

El diálogo existencial yo-tú (horizontal) para promover el darse cuenta refuerza la autorregulación organísmica sin buscar un cambio en el carácter que se ajuste a algún patrón de salud mental apegada a la psicoterapia Gestalt (Yontef, 1995). Cuando el paciente se da cuenta por sí mismo cómo interrumpe su autorregulación adquiere también la capacidad para aprender a cómo no interrumpirse, es decir, retomar el flujo de su autorregulación organísmica y concluir sus asuntos inconclusos (Perls, 1976). Durante el diálogo el psicoterapeuta retroalimenta o reafirma la capacidad de autorregulación organísmica del paciente (Spagnuolo, 2011). "En una autorregulación organísmica dialogal, la otra persona está contactada y sigue siendo una persona independiente, aunque aspectos de esa persona independiente, de ese otro, son asimilados en uno mismo" (Yontef, 1995, p. 37). 
El método dialogal de Yontef se basa en la relación dialogal Yo-Tú o Existencialismo Dialogal de Buber (Yontef, 1995). Las cualidades que definen el contacto del psicoterapeuta en la interrelación psicoterapéutica son: Inclusión, Presencia, Compromiso con el Diálogo, No explotación y Vivir la Relación, las cuales pueden observarse de forma aislada y también simultánea. La Inclusión consiste en comprender desde sí mismo, aceptar y respetar la experiencia fenomenológica del paciente. La Presencia radica en mostrarse genuino, como ser humano, y expresarse sin reservas. En el Compromiso con el Diálogo el encuentro psicoterapéutico es espontáneo, libre de expectativas. La No Explotación se distingue por una relación psicoterapéutica entre personas iguales, es decir considerar que el paciente es capaz de autodeterminarse. La Explotación se da cuando el paciente es tratado como un medio para cumplir un fin o un objetivo; hay desigualdad en el lenguaje al utilizar términos que el paciente no comprenda si la relación es vertical; el psicoterapeuta no cumple plenamente con su responsabilidad profesional; o no se establece un contexto relacional ético de trabajo. Vivir la Relación consiste en entregarse a la experiencia que surge en el momento, expresarse emocionalmente incluso sin palabras, así como proponer experimentos con la única intención de expandir el darse cuenta (Yontef, 1995).

\section{HABILIDADES PSICOTERAPÉUTICAS HUMANISTAS}

Para esta investigación se consideraron como habilidades psicoterapéuticas humanistas las características del contacto de la relación dialogal yo-tú del modelo dialogal de Yontef (1995) y las acciones vinculadas a la metodología humanista Gestalt para promover el darse cuenta y la autorregulación organísmica (mencionadas previamente).

\section{Cuestionario de habilidades psicoterapéuticas humanistas}

Se diseñó un cuestionario de habilidades psicoterapéuticas humanistas asociadas a los principios teórico-metodológicos de la psicoterapia Gestalt con el propósito de usarlo como un instrumento de evaluación de la frecuencia en que se emplean las habilidades psicoterapéuticas humanistas en la práctica. Se espera que pueda ser usado en un futuro para organizar y orientar los procesos de formación y supervisión psicoterapéutica de especialistas con enfoque humanista Gestalt.

En el diseño de la escala, se consideraron dos características particulares: 1) todas las preguntas fueron indicadores de medir habilidad y 2) las respuestas de cada sujeto se sumaron en un total que indica dónde se encuentra o cuánto tiene de la variable o características que pretendemos medir (Muñiz, 1996).

Aiken (1996), Anastasi y Urbina (1998), Muñiz (2000) y Cohen y Swerdlik (2006), señalan una serie de lineamientos y recomendaciones para la construcción de cada reactivo:

1. Disponer de otras fuentes de información sobre construcción de cuestionarios y escalas. Elegir la forma de respuesta al reactivo, es decir, que pueden variar desde respuestas dicotómicas con un sí y no o de tipo escala Likert que va de nada o mucho, entre otras formas de respuesta.

2. Evitar cuestionarios muy largos, lo que se pide es que la longitud sea razonable, teniendo en cuanta quiénes y en qué situación lo van a responder, limitándose a obtener la información que realmente interesa rescatar.

3. Evitar preguntar lo mismo más de una vez o lo que ya se sabe.

4. Evitar preguntas que requieren ordenar y de respuesta abierta.

5. Tener un plan inicial claro de la información que interesa recoger, hay que evitar hacer preguntas inútiles.

6. Que las oraciones sean cortas.

7. Preferentemente que los reactivos no lleven doble negación. 
8. Que los reactivos no tengan más de una idea a la vez.

9. Que sean afirmaciones positivas.

10. Que los reactivos sean claros y contextualizados a la población.

Por otro lado, es importante que las preguntas de identificación personal en cuestionarios y escalas vayan siempre al comienzo. Se distinguen tres tipos de datos: 1) Datos dicotómicos: sólo dos categorías de respuesta, sí o no, etcétera. Estos datos se codifican con unos y ceros. 2) Datos continuos que expresan una cantidad, o al menos un orden, se codifican con números originales, como puede ser curso y edad, también los datos que expresan grado de acuerdo, frecuencia, etcétera; es frecuente agruparlos en intervalos que se numeran correlativamente. 3) Datos nominales o cualitativos con más de dos respuestas para escoger una; son categorías de clasificación.

En el apartado sobre información personal conviene: 1) Mantener el anonimato del que responde para garantizar la sinceridad en las respuestas y también es un requerimiento que marca el código ético del psicólogo. Guardar la confidencialidad del participante. 2) No hacer más preguntas de las necesarias. 3) Evitar la opción otras. Los datos de información personal servirán para escribir la muestra y también para hacer análisis adicionales. Por lo tanto se requiere que el diseño del instrumento cuente con la validez de acuerdo con el grado para el que fue diseñada (Aiken, 1996; Anastasi \& Urbina, 1998; Cohen \& Swerdlik, 2006; Verdugo, Lucero, \& Alveano, 2006).

\section{METODOLOGÍA}

Con un tipo de estudio cuantitativo descriptivo con temporalidad transversal, el objetivo del presente estudio ha sido conocer los indicadores de fiabilidad de la Escala de Habilidades Psicoterapéuticas Humanistas (ehph), considerando el sistema de validación de jueceo de especialistas y de constructo a partir del análisis teórico. La ehph es una escala Likert con 32 ítems de autorreporte sobre la frecuencia del uso de las habilidades psicoterapéuticas humanistas con enfoque Gestalt en los procesos psicoterapéuticos, con opciones de respuesta del 1 al 5: (1) Casi nunca; (2) Ocasionalmente; (3) Algunas veces; (4) Frecuentemente; y (5) Casi siempre.

El instrumento fue elaborado por los autores siguiendo lineamientos de construcción de instrumentos de Davis citado en García Meraz (2010), el cual se sometió a un jueceo en el que participaron cuatro expertos en psicoterapia Gestalt, uno local, dos nacionales y otro internacional. La retroalimentación se realizó vía correo electrónico y mediante llamadas telefónicas. A partir de este jueceo se reelaboraron algunos de los 25 ítems originales relacionados con la metodología de la psicoterapia humanista Gestalt para promover la autorregulación organísmica dialogal (Kepner, 1999; Yontef, 1995; Zinker, 2003).

Una vez reconstruido se envió una invitación vía correo electrónico a 98 psicoterapeutas de alrededor de 200 que han cursado un posgrado en psicoterapia humanista en la región. Se utilizó una muestra no probabilística y dirigida ya que no se pretendía contar con una representatividad de los psicoterapeutas con estudios de posgrado (Hernández-Sampieri, Fernández-Collado, \& Baptista-Lucio, 2014). El criterio de selección de los participantes fue que hubieran concluido estudios de maestría y estuvieran activos en su práctica psicoterapéutica con enfoque humanista, con al menos un año de experiencia sin importar el ámbito (institucional, social o privado). Respondieron 51 participantes, lo que es conocido como muestreo no probabilístico por sujetos voluntarios (García Meraz, 2010), a quienes se les aplicó la ehph, incluyendo una carta de consentimiento informado. Se buscó, que por una parte el instrumento facilite la identificación de habilidades psicoterapéuticas en psicoterapeutas con orientación humanista en servicio.

Durante el piloteo se analizaron los 51 instrumentos capturados en el programa Estadístico para las Ciencias Sociales spss y mediante el análisis factorial exploratorio se lograron identificar los seis factores asociados a las fases del ciclo de la autorregulación del organismo (cao) de Zinker o ciclo de la experiencia 
de la psicoterapia Gestalt, con la aclaración de que fueron agrupados algunos de ellos: el factor sensación y el factor consciencia se agruparon como el factor darse cuenta, mientras el factor retirada se conjugó con conductas éticas del psicoterapeuta que promueven la independencia y el bienestar del paciente para quedar como el factor asimilación. Se establecieron cinco subescalas o dimensiones validadas del instrumento ehph como siguen:

1. Darse Cuenta (11 ítems). Habilidades para estimular el darse cuenta del paciente (Sensación y consciencia de las fases del cao).

2. Contacto (8 ítems). Habilidades para acentuar el contacto del paciente (fase de contacto del cao).

3. Acción (6 ítems). Habilidades para promover la toma de acciones del paciente (fase de acción del cao).

4. Autodeterminación (4 ítems). Habilidades para reconocer y respetar la responsabilidad del paciente sobre sus decisiones y elecciones (fase de movilización de energía y excitación del cao).

5. Asimilación (3 ítems). Habilidades para posibilitar el bienestar y la independencia del paciente (fase de retirada del cao).

La evaluación resultó en la obtención de un índice general de habilidades psicoterapéuticas surgido por la suma total de los 32 reactivos indicando tres rangos: (1) habilidades inadecuadas de 35 a 77, medias de 78 a 120 y habilidades adecuadas de 121 a 160. Asimismo, el factor 1 Darse Cuenta se consideró por rangos por intervalo en: inadecuado (11-24), medio (25-39) y adecuado (40-55). El factor 2 Contacto (inadecuado 8-18, medio 19-29, adecuado 30-40); el factor 3 Acción (inadecuado 6-14, medio 15-21, adecuado 22-30); el factor 4 Autodeterminación (inadecuado 4-9, medio 10-14, adecuado 15-20) y el factor 5 Asimilación (inadecuado 3-7, medio 8-11 y adecuado 12-15).

\section{RESULTADOS}

La ehph obtuvo un alpha de Cronbach total de $a=.865$. Con un $54.58 \%$ del total de varianza explicada en 5 factores con adecuación de muestreo pertinente $(\mathrm{kmo}=.624$, Bartlett JiCuadrada $=879.624, \mathrm{gl}=496$, Sig.=.000). Lo cual permitió identificar adecuada consistencia interna como factor de confiabilidad del instrumento según la Tabla 1, 1a, 1b. Se observó además que el Factor 1 Darse Cuenta obtuvo una carga factorial de $\mathrm{CF}=5.17$ con $16.2 \%$ de varianza y alpha de a $=.863$; el factor 2 Contacto logró una carga factorial de $\mathrm{CF}=4.07(12.7 \%)$ con $\mathrm{a}=.797$; el factor 3 Acción con $\mathrm{CF}=3.6(11.3 \%, \mathrm{a}=.810)$ y el factor 4 Autodeterminación $(\mathrm{CF}=2.3,7.2 \%, \mathrm{a}=.615)$. El factor 5 Asimilación obtuvo una carga factorial de $\mathrm{CF}=2.29$ con $7.1 \%$ pero con un alpha de $a=.309$, que en esta última dimensión se cuenta con un índice de baja consistencia interna. 
TABLA 1

Análisis factorial exploratorio de la Escala de Habilidades Psicoterapéuticas Humanistas

\begin{tabular}{|c|c|c|c|c|c|c|}
\hline \multirow[b]{2}{*}{ Factores } & \multirow[b]{2}{*}{ Items } & \multicolumn{5}{|c|}{ Componentes } \\
\hline & & 1 & 2 & 3 & 4 & 5 \\
\hline \multirow{13}{*}{$\begin{array}{l}\text { Darse Cuenta } \\
C F=5.17(16.2 \%)\end{array}$} & $\begin{array}{l}2 \text { Propongo un experimento para que mi paciente } \\
\text { se dé cuenta de sí misma(o). }\end{array}$ & 0.799 & & & & \\
\hline & $\begin{array}{l}32 \text { Le propongo a mi paciente experimentos para } \\
\text { fomentar su darse cuenta y no para que cambie. }\end{array}$ & 0.763 & & & & \\
\hline & $\begin{array}{l}3 \text { Promuevo que mi paciente se dé cuenta cómo } \\
\text { interrumpe su autorregulación sin forzar algún } \\
\text { cambio. }\end{array}$ & 0.749 & & & & \\
\hline & $\begin{array}{l}19 \text { Me enfoco en lo que va surgiendo entre mi } \\
\text { paciente y yo sin buscar otro objetivo más que el } \\
\text { darse cuenta. }\end{array}$ & 0.728 & & & & \\
\hline & $\begin{array}{l}17 \text { Propongo un experimento para que mi } \\
\text { paciente se dé cuenta de sus recursos. }\end{array}$ & 0.714 & & & & \\
\hline & $\begin{array}{l}1 \text { Le describo a mi paciente lo que observo de él } \\
\text { (ella) para fomentar su darse cuenta, y no lo que } \\
\text { podría o debería ser. } \\
6 \text { La comunicación entre mi paciente y yo no } \\
\text { es únicamente con palabras, también incluye } \\
\text { movimiento, danza, sonidos, melodías, canciones } \\
\text { o expresiones gráficas. }\end{array}$ & 0.620 & & & & \\
\hline & $\begin{array}{l}25 \text { Le pregunto a mi paciente qué necesita hasta } \\
\text { que haya encontrado el significado de lo que } \\
\text { siente. }\end{array}$ & 0.526 & & & & \\
\hline & $\begin{array}{l}18 \text { Identifico con claridad cómo mi paciente } \\
\text { obstaculiza su autorregulación. }\end{array}$ & 0.520 & & & & \\
\hline & $\begin{array}{l}31 \text { Promuevo la expresión emocional de mi } \\
\text { paciente después de que haya encontrado el } \\
\text { significado de su sentimiento. }\end{array}$ & 0.481 & & & & \\
\hline & $\begin{array}{l}11 \text { Promuevo que mi paciente relate historias } \\
\text { únicamente para que identifique qué es lo que le } \\
\text { hace sentir su relato. }\end{array}$ & 0.436 & & & & \\
\hline & $29 \mathrm{Me}$ intereso en lo que comunica mi paciente. & & 0.778 & & & \\
\hline & $\begin{array}{l}13 \text { Acepto las diferencias entre la subjetividad de } \\
\text { mi paciente y la mía. }\end{array}$ & & 0.683 & & & \\
\hline & $\begin{array}{l}28 \text { Muestro mi naturaleza humana al paciente, sin } \\
\text { aparentar ni asumir poses de superioridad. }\end{array}$ & & 0.665 & & & \\
\hline
\end{tabular}

Alpha total $\mathrm{a}=.865 .54 .58 \%$ Total de varianza explicada en 5 factores

$(\mathrm{KMO}=.624$, Bartlett JiCuadrada=879.624, gl=496, Sig. $=.000)$

Método de extracción empleado: análisis de componentes principales.

Método de rotación empleado: Varimax con normalización Kaiser. 
TABLA 1 (CONT.)

Análisis factorial exploratorio de la Escala de Habilidades Psicoterapéuticas Humanistas

\begin{tabular}{|c|c|c|c|c|c|c|}
\hline \multirow[b]{2}{*}{ Factores } & \multirow[b]{2}{*}{ Items } & \multicolumn{5}{|c|}{ Componentes } \\
\hline & & 1 & 2 & 3 & 4 & 5 \\
\hline \multirow{7}{*}{$\begin{array}{l}\text { Contacto } \\
\mathrm{CF}=4.07(12.7 \%)\end{array}$} & 24 Soy espontánea(o) con mi paciente. & & 0.650 & & & \\
\hline & $\begin{array}{l}30 \text { Fomento la expresión de los sentimientos y } \\
\text { emociones de mi paciente. }\end{array}$ & & 0.623 & & & \\
\hline & 22 Respeto los silencios de mi paciente. & & 0.473 & & & \\
\hline & $\begin{array}{l}20 \text { Empatizo con mi paciente, sin perder mi } \\
\text { autonomía, mi yo. }\end{array}$ & & 0.465 & & & \\
\hline & 26 Adecúo mi lenguaje al lenguaje de mi paciente. & & 0.423 & & & \\
\hline & $\begin{array}{l}21 \text { Promuevo que mi paciente tome acciones en su } \\
\text { vida a partir de lo que ha aprendido en su proceso } \\
\text { psicoterapéutico. }\end{array}$ & & & 0.790 & & \\
\hline & $\begin{array}{l}12 \text { Le doy información a mi paciente que le facilite } \\
\text { comprenderse a sí misma(o) y a su mundo. }\end{array}$ & & & 0.722 & & \\
\hline Acción & 10 Estimulo la espontaneidad de mi paciente. & & & 0.684 & & \\
\hline \multirow[t]{4}{*}{$C F=3.6(11.3 \%)$} & $\begin{array}{l}23 \text { Apoyo las acciones de mi paciente que } \\
\text { favorecen su autorregulación. }\end{array}$ & & & 0.638 & & \\
\hline & $\begin{array}{l}16 \text { Le hago saber a mi paciente las fortalezas que } \\
\text { descubro en él (ella). }\end{array}$ & & & 0.627 & & \\
\hline & $\begin{array}{l}14 \text { Exploro con preguntas abiertas (qué, quién, } \\
\text { cuál, dónde, cómo, etcétera) para recabar } \\
\text { información precisa de mi paciente. }\end{array}$ & & & 0.433 & & \\
\hline & $\begin{array}{l}9 \text { Asumo que mi paciente es responsable y capaz } \\
\text { de elegir por sí mismo(a). }\end{array}$ & & & & 0.593 & \\
\hline
\end{tabular}

Alpha total $a=.865 .54 .58 \%$ Total de varianza explicada en 5 factores $(\mathrm{KMO}=.624$, Bartlett JiCuadrada $=879.624$, gl=496, Sig. $=.000)$

Método de extracción empleado: análisis de componentes principales.

Método de rotación empleado: Varimax con normalización Kaiser.

TABLA 1 (CONT.)

Análisis factorial exploratorio de la Escala de Habilidades Psicoterapéuticas Humanistas

\begin{tabular}{|c|c|c|c|c|c|c|}
\hline \multirow[b]{2}{*}{ Factores } & \multirow[b]{2}{*}{ Items } & \multicolumn{5}{|c|}{ Componentes } \\
\hline & & 1 & 2 & 3 & 4 & 5 \\
\hline \multirow[t]{4}{*}{$\begin{array}{l}\text { Autodeterminación } \\
\mathrm{CF}=2.3(7.2 \%)\end{array}$} & $\begin{array}{l}4 \text { Respeto la manera en que mi paciente se vive a } \\
\text { sí mismo(a). }\end{array}$ & & & & 0.569 & \\
\hline & 7 Acepto incondicionalmente a mi paciente. & & & & 0.541 & \\
\hline & 27 Confío en mi paciente. & & & & 0.344 & \\
\hline & $\begin{array}{l}5 \text { Me apego a un código ético que promueva el } \\
\text { bienestar de mi paciente. }\end{array}$ & & & & & 0.575 \\
\hline \multirow[t]{2}{*}{$\begin{array}{l}\text { Asimilación } \\
\mathrm{CF}=2.29(7.1 \%)\end{array}$} & $\begin{array}{l}15 \text { Evito dramatizar las fantasías de mi paciente } \\
\text {-del pasado o del futuro- para experimentarlas } \\
\text { como si estuvieran sucediendo en el presente. }\end{array}$ & & & & & 0.560 \\
\hline & $\begin{array}{l}8 \text { Fomento que mi paciente aprenda a quedarse } \\
\text { con lo nutritivo y desechar lo que no es nutritivo } \\
\text { de las experiencias. }\end{array}$ & & & & & 0.554 \\
\hline $\mathrm{N}=51$ & Alpha & 0.863 & 0.797 & 0.810 & 0.615 & 0.309 \\
\hline
\end{tabular}

Alpha total $a=.865 .54 .58 \%$ Total de varianza explicada en 5 factores

$(\mathrm{KMO}=.624$, Bartlett JiCuadrada $=879.624, \mathrm{gl}=496$, Sig. $=.000)$ 
Método de extracción empleado: análisis de componentes principales.

Método de rotación empleado: Varimax con normalización Kaiser.

Por otro lado, el 63\% (32) de los participantes fueron mujeres mientras que el 37\% (19) hombres, con una media de edad de $\mathrm{x}=41.6$ (DS=10.3) entre 26 y 78 años. El 43\% (22) labora en Consultorio privado, 14\% (7) en Institución pública, así como el 21\% (11) en ambos, además del 14\% (7) labora en consulta privada y en una Organización No Gubernamental (ong) y $8 \%$ (4) sólo en ong, con un promedio de $\mathrm{x}=7.8$ (DS= 4.7) años de experiencia siendo el 43\% (22) con menos de tres años de experiencia, 31\% (16) de 4 a 6 años y el 26\% (13) más de seis años. 66\% (39) cuentan con estudios completos de Maestría y el 24\% (12) con Doctorado en curso, siendo el 61\% (31) egresados del nivel de Licenciatura de la Universidad Autónoma de Ciudad Juárez [UACJ] y el 40\% (10) de otra institución educativa; mientras que a nivel Maestría el 80\% (41) reportaron haber egresado de la Maestría en Psicoterapia Humanista y Educación para la Paz y el 20\% (10) de otro programa de posgrado diferente. Asimismo, el 77\% (39) son psicólogos y el 23\% (12) cuentan con otra licenciatura sin ser psicólogos, 2 de ellos egresaron de Economía, otros 2 de Educación, 2 de Ingeniería y 1 de cada una de las siguientes Licenciaturas: Agronomía, Contaduría, Enfermería, Nutrición, Sociología, y Relaciones Comerciales.

Se observó que en la Escala total de Habilidades Psicoterapéuticas se obtuvo un promedio de $\mathrm{x}=135.4$ $(\mathrm{ds}=10.9)$ siendo el 16\% (8) con medianas habilidades mientras que el 84\% (43) consideradas como habilidades psicoterapéuticas adecuadas. Asimismo, 65\% mostró habilidades adecuadas en la categoría de Darse Cuenta y $33 \%$ medianas, observando que en sólo un psicoterapeuta (2\%) fue inadecuada ( $x=42$, DS=7). El 94\% consideró realizar habilidades adecuadas para promover el Contacto y la Acción, el 96\% de Autodeterminación y en cuanto a la Asimilación, el $57 \%$ se consideró con habilidades adecuadas, $41 \%$ medianas y el $2 \%$ (un psicoterapeuta) con habilidad inadecuada (ver Tabla 2).

TABLA 2

Descriptivos y frecuencias de factores

\begin{tabular}{lccccc}
\multicolumn{1}{c}{$\mathrm{N}=5 \mathbf{5 1}$} & Media & DS & Inadecuado & Medio & Adecuado \\
Darse Cuenta & 42,0 & 7,0 & $2 \%(1)$ & $33 \%(17)$ & $65 \%(33)$ \\
Contacto & 36,7 & 3,2 & & $6 \%(3)$ & $94 \%(48)$ \\
Acción & 26,5 & 3,1 & & $6 \%(3)$ & $94 \%(48)$ \\
Autodeterminación & 18,4 & 1,7 & & $4 \%(2)$ & $96 \%(49)$ \\
Asimilación & 11,8 & 1,6 & $2 \%(1)$ & $41 \%(21)$ & $57 \%(29)$ \\
\hline Habilidades Psicoterapéuticas & 135,4 & 10,9 & & $16 \%(8)$ & $84 \%(43)$ \\
\hline
\end{tabular}

En el análisis de varianza y de diferencia de medias, no se encontró discrepancia estadísticamente significativa por sexo, grado académico, institución donde labora aunque las tendencias indicaron habilidades psicoterapéuticas ligeramente mayores en hombres $(x=137.6)$ que en las mujeres $(x=134.1)$ y en cada dimensión excepto en Asimilación donde las mujeres $(\mathrm{x}=12.1)$ reportaron mayores habilidades que los hombres $(\mathrm{x}=11.1)$. También, en aquellos psicoterapeutas que cursaron maestría en relación con los doctorantes $(\mathrm{x}=136.3, \mathrm{x}=133.7)$ excepto en Asimilación donde los doctorantes reportaron mayores habilidades que los egresados de maestría $(\mathrm{x}=11.8, \mathrm{x}=12.0)$. Los psicoterapeutas que laboran sólo en ong mantuvieron habilidades altas $(x=141.5)$ con relación a los que laboran en ong y en consultorio privado $(x=136.9)$, a los que laboran en institución pública $(x=136.1)$, a los que trabajan en institución privada $(x=135)$ y de los que laboran en institución pública y en consultorio privado $(x=132.6)$ según la Tabla 3. 
TABLA 3

Descriptivos factores por sexo, grado académico e institución donde labora

\begin{tabular}{|c|c|c|c|c|c|c|c|c|c|}
\hline & \multirow{2}{*}{\multicolumn{2}{|c|}{ Sexo }} & \multirow{2}{*}{\multicolumn{2}{|c|}{ Grado académico }} & \multirow{2}{*}{\multicolumn{5}{|c|}{ Institución laboral }} \\
\hline & & & & & & & & & \\
\hline & $\begin{array}{l}\text { Mujer } \\
(\mathrm{n}=32)\end{array}$ & $\begin{array}{l}\text { Hombre } \\
(n=19)\end{array}$ & $\begin{array}{c}\text { Maestría } \\
(n=34)\end{array}$ & $\begin{array}{l}\text { Doctorante } \\
\quad(n=17)\end{array}$ & $\begin{array}{c}\text { Pública } \\
(n=7)\end{array}$ & $\begin{array}{l}\text { Privado } \\
(n=22)\end{array}$ & $\begin{array}{l}\text { Púb-Priv } \\
(n=11)\end{array}$ & $\begin{array}{c}\text { ONG y } \\
\text { privada } \\
(n=7)\end{array}$ & $\begin{array}{l}\text { ONG } \\
(n=4)\end{array}$ \\
\hline Darse Cuenta & 41,1 & 43,6 & 43,2 & 39,7 & 38,9 & 42,8 & 40,6 & 43,6 & 44,8 \\
\hline Contacto & 36,5 & 36,9 & 36,8 & 36,4 & 38,1 & 36,3 & 36,0 & 36,9 & 37,5 \\
\hline Acción & 26,0 & 27,3 & 26,2 & 26,9 & 27,4 & 26,3 & 25,5 & 26,9 & 27,8 \\
\hline Autodeterminación & 18,5 & 18,3 & 18,3 & 18,6 & 19,4 & 17,9 & 18,7 & 18,0 & 19,3 \\
\hline Asimilación & 12,1 & 11,5 & 11,8 & 12,0 & 12,3 & 11,7 & 11,8 & 11,6 & 12,3 \\
\hline $\begin{array}{l}\text { Habilidades Psico- } \\
\text { terapéuticas }\end{array}$ & 134,1 & 137,6 & 136,3 & 133,7 & 136,1 & 135,0 & 132,6 & 136,9 & 141,5 \\
\hline
\end{tabular}

Asimismo, los psicoterapeutas formados en la uacj (61\%) reportaron mayores habilidades ( $x=136.1)$ que los que se formaron en otras instituciones $(\mathrm{x}=134.3)$ sin diferencia estadística significativa independientemente del programa educativo ni de si son o no psicólogos. Otra tendencia sin significancia estadística fue que los psicoterapeutas que tienen de 3 a 6 años de experiencia reportaron mayores habilidades $(\mathrm{x}=138.1)$ que los que tienen más de 6 años $(\mathrm{x}=135.5)$ y los que tienen menos de 3 años $(\mathrm{x}=133.4)$ de experiencia (ver Tabla 4).

TABLA 4

Descriptivos factores por formación y experiencia

\begin{tabular}{|c|c|c|c|c|c|c|c|c|}
\hline \multicolumn{2}{|c|}{ Formación } & \multicolumn{2}{|c|}{ Programa } & \multicolumn{2}{|c|}{ Psicólogos-otros } & \multicolumn{3}{|c|}{ Experiencia } \\
\hline$\underset{(n=31)}{\text { UACJ }}$ & $\begin{array}{c}\text { Otros } \\
(n=20)\end{array}$ & $\begin{array}{l}\text { MPHEP } \\
(n=41)\end{array}$ & $\begin{array}{c}\text { Otros } \\
(n=10)\end{array}$ & $\begin{array}{l}\text { Psicólogos } \\
(n=39)\end{array}$ & $\begin{array}{l}\text { No psicólo- } \\
\text { gos }(n=12)\end{array}$ & $\begin{array}{c}0-3 \\
\text { años } \\
(n=22)\end{array}$ & $\begin{array}{c}3 \text { a } 6 \\
\text { años } \\
(n=16)\end{array}$ & $\begin{array}{c}\text { Más } \\
\text { de } 6 \\
\text { años } \\
(n=13)\end{array}$ \\
\hline 41,8 & 42,4 & 42,6 & 39,6 & 41,9 & 42,6 & 41,6 & 43,9 & 40,6 \\
\hline 37,3 & 35,7 & 36,5 & 37,1 & 36,7 & 36,4 & 36,1 & 37,3 & 36,8 \\
\hline 26,7 & 26,2 & 26,1 & 27,9 & 26,7 & 25,8 & 25,9 & 26,6 & 27,3 \\
\hline 18,7 & 18,0 & 18,4 & 18,5 & 18,4 & 18,4 & 18,1 & 18,4 & 18,9 \\
\hline 11,7 & 12,1 & 11,7 & 12,4 & 11,8 & 12,0 & 11,8 & 11,9 & 11,9 \\
\hline 136,1 & 134,3 & 135,4 & 135,5 & 135,5 & 135,3 & 133,4 & 138,1 & 135,5 \\
\hline
\end{tabular}

Las habilidades psicoterapéuticas estuvieron fuertemente relacionaas con las sub dimensiones de: Darse Cuenta $\left(\mathrm{R}=.790^{* *}\right)$, Contacto $\left(\mathrm{r}=.790^{* *}\right)$ y Acción $\left(\mathrm{r}=.701^{* *}\right)$; medianamente con el Autodeterminación $\left(\mathrm{r}=.404^{* *}\right)$ y sin correlación con la Asimilación. Se identificó relación significativa entre el Darse Cuenta con el Contacto $\left(\mathrm{r}=284^{*}\right)$ y con la Acción $(\mathrm{r}=.018)$, que a su vez presenta relación con la Autodeterminación $\left(\mathrm{r}=.319^{*}\right)$ y esta última presentó relación significativa con la dimensión de Asimilación $\left(\mathrm{r}=.342^{*}\right)$. Todas estas asociaciones se presentaron en forma estadísticamente positiva, es decir, que a medida que aumenta el desarrollo de una habilidad, también aumentan las demás según la Tabla 5. 
TABLA 5

Correlaciones Pearson entre factores

\begin{tabular}{|c|c|c|c|c|c|}
\hline & Contacto & Acción & Elección & Etica & Habilidades Psicoterapéuticas \\
\hline Darse Cuenta & $.284 *(.044)$ & $.331 *(.018)$ & $-0.018(.901)$ & $-0.15(.293)$ & $.790^{* *}(.000)$ \\
\hline Contacto & & $.499 * *(.000)$ & $.410 * *(.003)$ & $0.232(.101)$ & $.709 * *(.000)$ \\
\hline Acción & & & $.319 *(.022)$ & $0.09(.532)$ & $.701^{* *}(.000)$ \\
\hline Autodeterminación & & & & $.342 *(.014)$ & $.404^{* *}(.003)$ \\
\hline Asimilación & & & & & $0.19(.162)$ \\
\hline
\end{tabular}

\footnotetext{
${ }^{* *}$ La correlación resultó significativa en el nivel 0,01 (bilateral).

${ }^{*}$ La correlación resultó significativa en el nivel 0,05 (bilateral).
}

\section{DISCUSIÓN}

Los integrantes de la sociedad merecen una atención de alta calidad para atender su salud mental. Los psicoterapeutas que tienen oportunidad de atenderlos requieren de una formación profesional basada en métodos actuales científicamente comprobados. La formación del psicoterapeuta es constante, no basta con la formación que reciben en las instituciones académicas, sino que requiere de una actualización permanente. Los instrumentos de evaluación de las habilidades psicoterapéuticas son un medio para reconocer su capacidad profesional para atender a sus pacientes y afianzarla, además, que posibilitan obtener una perspectiva que les permita establecer estrategias de mejora.

Los ítems que componen la Escala de Habilidades Psicoterapéuticas Humanistas coinciden con las características de la psicoterapia Gestalt definidas en el prototipo de Case (2011) y con las prácticas de los conceptos básicos de la Escala de Fidelidad de la Terapia Gestalt (Fogarty, 2015). Este instrumento resultó con una buena consistencia interna con $\mathrm{a}=.865$ con un adecuado análisis factorial apto para ser válido y confiable en la medición de la frecuencia del uso de las habilidades psicoterapéuticas humanistas (Darse Cuenta, Contacto, Acción, Autodeterminación y Asimilación) en la práctica de procesos psicoterapéuticos que tienen lugar en la zona de Ciudad Juárez, Chihuahua.

El $84 \%$ de psicoterapeutas de la región mostró tener habilidades psicoterapéuticas adecuadas mientras el 16\% restante habilidades medianas. El 65\% obtuvo habilidades adecuadas en el darse cuenta, el 33\% medianas y solamente un psicoterapeuta inadecuadas (2\%). Mediante estas habilidades el psicoterapeuta acompaña al paciente para que identifique la necesidad imperante del organismo y las posibilidades del campo organismo-ambiente para satisfacerlas. El 96\% cuenta con habilidades adecuadas en Autodeterminación, con estas habilidades el psicoterapeuta apoya al paciente para que tome sus propias decisiones. Por otra parte el 94\% muestra habilidades de Contacto y Acción adecuadas. Las habilidades de Contacto favorecen el intercambio entre el organismo y su ambiente, mientras las de Acción fomentan las expresiones físicas que lleven al contacto con el posible satisfactor. En cuanto a la habilidad de Asimilación, el 57\% tiene un nivel adecuado, $41 \%$ mediano y el $2 \%$ (un psicoterapeuta) inadecuado. Mediante estas habilidades el psicoterapeuta fomenta la autonomía y bienestar del paciente (Case, 2011; Castanedo, 1999; Kepner, 1999; Perls et al., 2003; Zinker, 2003).

Se espera que la ehph pueda ser utilizada en procesos de formación y supervisión de profesionales en esta área como guía para identificar tanto los aspectos que pueden ser reafirmados como los que pueden desarrollarse más ampliamente para proporcionar un servicio cada vez mejor a los pacientes. 


\section{LIMITACIONES}

El tamaño de la muestra pudiera limitar el panorama sobre las habilidades que los psicoterapeutas activos de la región emplean en su práctica cotidiana. También se puede realizar un análisis más detallado que muestre las similitudes y diferencias entre los ítems de la Escala de Habilidades Psicoterapéuticas Humanistas y las características del prototipo de Case y los comportamientos de la Escala de la Fidelidad de la Terapia Gestalt que de lugar a ampliar la cantidad de ítems.

\section{POSIBILIDADES FUTURAS DE INVESTIGACIÓN}

Para investigaciones por venir, se puede ampliar la muestra así como incluir más ítems con el propósito de aumentar la consistencia interna del factor de Asimilación, así como la fiabilidad del instrumento.

\section{REFERENCIAS}

Aiken, L. R. (1996). Tests psicológicos y evaluación. México: Prentice Hall.

Anastasi, A., \& Urbina, S. (1998). Tests psicológicos. México: Prentice Hall.

Bados, A., \& García, E. (2011). Habilidades terapéuticas. Barcelona, España: Universitat de Barcelona.

Bados, A., García, E., \& Fusté, A. (2002). Eficacia y utilidad clínica de la terapiapsicológica. International Journal of Clinical and Health Psychology, 2(3), 477-502.

Beitman, B., \& Yue, D. (2004). Psicoterapia: Programa de formación. Barcelona, España: Masson.

Casas, M. (2011). Técnicas expresivas: Su integración en las prácticas profesionales de los psicólogos egresados de la Universidad de la República (Tesis doctoral). Uruguay: Universidad de la República.

Case, M. (2011). Measuring processes in contemporary gestalt therapy. Development of gestalt therapy prototype and applications in research (Tesis doctoral). Oregon, US: Pacific University.

Castanedo, C. (1999). Terapia Gestalt. Enfoque centrado en el aqui y ahora. Barcelona, España: Herder.

Castanedo, C., \& Munguía, G. (2011). Diagnóstico, intervención e investigación en psicología humanista. Madrid, España: Editorial CSS.

Cohen, R. J., \& Swerdlik, M. E. (2006). Pruebas y evaluación psicológicas. Introducción a las pruebas y a la medición. México: McGraw-Hill.

Fagan, J., \& Shepherd, I. (1973). Teoría y técnica de la psicoterapia guestáltica. Buenos Aires, Argentina: Amorrortu Editores.

Ferro, R., \& Vives M. (2004). Análisis de los conceptos de efectividad, eficacia y eficiencia en psicología. Panace, 5(16), 97-99.

Fogarty, M. (2015). Creating a fidelity scale for gestalt therapy. Gestalt Journal of Australia and New Zealand, 11(2), 39. ., Bhar, S., Theiler, S., \& O'Shea, L. (2016). What do Gestalt therapists do in the clinic? The expert consensus. British Gestalt Journal, 25(1), 32-41.

García Meraz, M. (2010). Medición en psicología: Del individuo a la interacción. Pachuca, México: Universidad Autónoma del Estado de Hidalgo.

Hernández-Sampieri, R., Fernández-Collado, C., \& Baptista-Lucio, P. (2014). Metodología de la investigación. México: McGraw-Hill.

Kepner, J. (1999). Proceso corporal. Un enfoque Gestalt para el trabajo corporal en psicoterapia. México: Editorial El Manual Moderno.

Marinho, M., Caballo, V., \& Silveira J. (2003). Cuestiones olvidadas en la terapia conductual: las habilidades del terapeuta. Revista Psicología Conductual, 11(1), 135-161. 
Muñiz, J. (1996). Psicometría. Madrid, España: Universitarias. . (2000). Teoría clásica de los tests. Madrid, España: Pirámide.

oms (2003). Quality improvement for mental health. who mental health policy and service guidance package - module 8. Ginebra: Organización Mundial de la Salud. Recuperado de https://www.who.int/mental_health/policy/se rvices/essentialpackage1v8/en/

(2005). Human resources and training in mental health. WHO mental health policy and service guidance package - module 9. Ginebra: Organización Mundial de la Salud. Recuperado de https://www.who.int/mental_health/ policy/services/essentialpackage1v9/en/

Paredes-Rivera, A. (2016). La urgencia de la evidencia en psicoterapia. Interacciones: Revista de Avances en Psicología, 2(1), 53-63. doi:10.24016/2016.v2n1.21

Perls, F. (1974). Sueños y existencia. Santiago, Chile: Editorial Cuatro Vientos.

. (1975). Dentro y Fuera del Tarro de la Basura. Santiago, Chile: Editorial Cuatro Vientos.

. (1976). El Enfoque guestáltico y testimonios de terapia. Santiago, Chile: Editorial Cuatro Vientos.

,Hefferline, R., \& Goodman, P. (2003). Terapia gestalt. Excitación y crecimiento de la personalidad humana. Madrid, España: Sociedad de Cultura Valle-Inclán.

rae (2019). Diccionario de la real academia española. España: Asociación de Academias de la Lengua Española.

Sánchez-Sosa, J. (Abril, 2012). Aspectos contemporáneos en la regulación de la psicoterapia y el consejo psicológico en México. Trabajo presentado en la conferencia del Congreso Nacional de la Asociación Mexicana de Psicología Clínica y de la Salud, Acapulco, México.

Spagnuolo, M. (2011). El ahora-para-lo-siguiente en psicoterapia. La psicoterapia de la Gestalt contada en la sociedad post-moderna. Madrid, España: Asociación Cultural Los Libros del ctp.

SS (2014). Programa de acción específico salud mental 2013-2014. México: Secretaría de Salud. Recuperado de https:/ /www.gob.mx/cms/uploads/attachment/file/11918/Salud_Mental.pdf

Taragano, F. (1974). Psicoanálisis gestáltico. Buenos Aires, Argentina: Editorial Paidós.

Verdugo Lucero, J., Ochoa Alcaraz, S., \& Alveano Hernández, J. (2006). Elaboración de cuestionarios. Colima, México: Universidad de Colima.

Winkler, M., Cáceres, C., Fernández, I., \& Sanhueza, J. (1989). Factores inespecíficos de la psicoterapia y efectividad del proceso terapéutico: Una sistematización. Revista Terapia Psicológica, 8(11), 34-40.

Yontef, G. (1995). Proceso y diálogo en Gestalt: Ensayos de terapia gestáltica. Santiago, Chile: Cuatro Vientos Editorial. Zinker, J. (2003). El proceso creativo en la terapia Gestalt. México: Editorial Paidós. 